\title{
INTRODUCTORY MICROMACHINING AND MEMS COURSE FOR GRADUATE AND UNDERGRADUATE STUDENTS
}

\author{
Jack W. Judy ${ }^{1}$ and Paulo S. Motta ${ }^{2}$ \\ ${ }^{1}$ Electrical Engineering Department, University of California, Los Angeles, Los Angeles, CA 90095 USA \\ ${ }^{2}$ Innovative Micro Technology, Goleta, CA 93117, USA
}

\begin{abstract}
An undergraduate-level course that provided an introduction to the fields of MEMS, with an emphasis on the processes used to microfabricate MEMS, was first developed at UCLA in 1998. It has served well to quickly ramp-up new MEMS graduate students that have lacked prior experience with MEMS. It has also given undergraduates and students even outside of the school of engineering, an opportunity to be exposed to micromachining and MEMS. A comprehensive, weekly, and hands-on laboratory session gave all students a greater appreciation for the capabilities and limitations of micromachining processes and MEMS devices. Due to excessive popularity and safety limitations on the number of students allowed into each lab session, the course was split into two separate courses: a lecture-only course and a laboratory course. Now students from nearly any background can learn about MEMS and could be the seeds of future uses of MEMS technology in their chosen discipline. These courses also serve as a spring board into the formal MEMS graduate program at UCLA, as well as into the MEMS industry.
\end{abstract}

\section{MOTIVATION AND BACKGROUND}

Since 1998, UCLA has offered introductory MEMS courses specifically designed for both undergraduate and graduate students. Up until that time, MEMS courses at universities were primarily, if not exclusively, offered at the graduate-student level. The motivation behind offering MEMS courses also at the undergraduate level was driven by the following: (1) the growing MEMS industry demanded a MEMS-aware/knowledgeable work force at all levels (Ph.D., M.S., and B.S.); (2) the expanding diversity of MEMS applications attracted students from many different backgrounds with no prior knowledge of micromachining; (3) the core material on MEMS fabrication is easily accessible to undergraduates (i.e., no upper-division prerequisites are truly required). With NSF Career-Award funding, a course titled "Introduction to Micromachining and MEMS" was designed to consist of two major components: a main lecture sequence and weekly hands-on laboratory sessions. Later, due to popularly and safety constraints, the course was split into two separate courses: a lecture-only course and a lab course.

An important issue in the teaching of micromachining and MEMS is the reality that the students taking the course will have very different backgrounds. For example, we found it common for graduate students from mechanical engineering to have no knowledge or experience with IC fabrication and other micromachining techniques. In addition, students from outside of engineering, such as those in the life sciences or medicine, will lack much of the engineering background that is typically taken for granted, and thus will also struggle in a micromachining and MEMS class taught at the graduate-level. This is particularly unfortunate, since the field of MEMS gains tremendously by the involvement of people from diverse backgrounds, because of the unique needs, applications, and abilities of their field.

We have found that an undergraduate course on micromachining and MEMS is an excellent instrument for addressing these issues. The level of the course makes it much easier to cover sufficient background material, which enables students with no knowledge of micromachining or MEMS to participate. Adding a laboratory component facilitates the solidification of this first-time learning experience. After taking this course, students from almost any background are able to go on to the graduate-level courses in MEMS with confidence.

It is well known that providing hands-on learning experiences is one of the most effective ways of teaching. This is particularly true when there are many details that are important, yet can easily be passed over in lecture or are quickly forgotten. Good examples of material like this are micromachining processes and MEMS. Describing a micromachining process or, for example, the stiction problem in MEMS, and actually experiencing them are two very different things. Clearly the hands-on lesson will be longer lasting and is also likely to provide additional unexpectedly valuable lessons.

\section{COURSE STRUCTURE AND DESIGN}

Initially, the lecture and laboratory components were integrated into one common course. However, to ease enrollment limitations (described later in the text) and to provide full credit to the students and instructors for their effort, the course was split into two courses: a lecture-only course (two 2-hour lectures/week) and a laboratory course, which consists of a weekly lab-oriented lecture ( 2 hours/week) and a weekly lab session (4 hours/week). Although potentially a large number of students may enroll solely in the lecture-only course $(\sim 100$ students in 2003$)$, enrollment in the lab course is greatly limited by safety concerns (max of 10 students in each of the 6 weekly lab sessions) and requires coenrollment in the lecture-only course. Due to UCLA being on a quarter system, instruction is carried out over 10-week quarters.

\section{A. Prerequisites}

Since this course is intentionally designed for students with almost any background, the prerequisites include a nominal exposure to college-level chemistry, calculus, and physics.

\section{B. Textbook}

There is no single textbook that is optimal for this undergraduate micromachining and MEMS course. Currently chapters from multiple texts [1-6] are compiled with selected conference and journal papers into a single course reader.

\section{Lecture Course}

The purpose of this lecture course is to provide both a broad perspective of the field in general and to instruct the students on the fundaments and theory involved in each of the major micromachining process technologies. The course begins by introducing the field of MEMS and provides examples to demonstrate its broad utility, stimulate interest, and promote a curiosity for how MEMS are made. After the basic concept of micromachining is introduced, vital micromachining processes are described. A week-by-week breakdown of the material covered in the lecture course is given below in Table 1. Weekly homework assignments are used to allow students to practice the lessons taught in lecture. 
Table 1. Outline of the lecture course.

\begin{tabular}{cl}
\hline Week & Lecture Material \\
\hline 1a & Introduction, Overview, and History of MEMS \\
1b & Applications of MEMS with Examples \\
2a & Process Flow and Crystallography \\
2b & Chemical Safety, Clean Rooms, Cleaning Procedures \\
3a & Mask Making, Layout, Photolithography Basics \\
3b & Photolithography Tools \\
4a & Wet Etching (Isotropic, Anisotropic) \\
4b & Wet Etching (Anisotropic) \\
5a & Vacuum Systems \\
5b & Midterm Exam \\
6a & Plasmas and Dry Etching (Plasma Etching) \\
6b & Dry Etching (RIE, Sputter Etching, Ion Milling) \\
7a & Physical Vapor Deposition (Evaporation, Sputtering) \\
7b & Diffusion \\
8a & Ion Implantation \\
8b & Thermal Oxidation \\
$9 \mathrm{a}$ & Chemical Vapor Deposition (LPCVD, PECVD) \\
9b & Electroplating and Design of Experiments (DOE) \\
10a & Design of Experiments (DOE), Process Integration \\
10b & Process Integration \\
\hline
\end{tabular}

Obviously, a 10-week quarter is not long enough to include a thorough discussion of advanced microfabrication processes (e.g., advanced lithography, thick-film lithography, soft lithography, DRIE, wafer bonding, electroless plating, photo-electro-chemical etching, chemical-mechanical polishing, stiction and release techniques, batch-assembly techniques, and the mechanical properties of thin-films). As a result, a follow-on graduate-level MEMS-fabrication course is offered at UCLA (EE M250A / MAE M280 / BME M250A: MEMS Fabrication), which is designed to cover these more advanced topics. MEMS-design issues are covered in the second graduate-level MEMS course offered at UCLA (EE M250B / MAE M282 / BME M250B: MEMS Design). To make up for the lack of advanced material in the undergraduate course described here, in each lecture the relevance of the material covered is briefly related to MEMS by the inclusion of pertinent examples taken from the MEMS literature.

\section{Laboratory Course}

The primary purpose of the laboratory course is to provide hands-on experience fabricating, testing, and characterizing MEMS, in a manner that compliments the content delivered in the lecture-only course. Specifically, the student gains hands-on experience in photolithography, etching (wet and dry surface and bulk micromachining), metal deposition (evaporation, sputtering, and electrodeposition), and several metrology tools (microscope, profilometer, probe station, four-point probe, and spectrometer). The devices that are fabricated as part of this laboratory include: accelerometers, thermal micoractuators, magnetic microactuators [7] and microsensors, fiber-optic switches, neural probes, and pressure sensors (Figs. 1 and 2). The fabrication process is described in a later sub-section.

Although there are 6 separate 10-student ( $\max$ ) laboratory sessions, there is a common lecture specifically for this lab course to insure the students are fully briefed and prepared for the upcoming lab sessions. This laboratory course is taught in the instructional wing of the UCLA Nanoelectronics Research Facility (Nanolab), known as the UCLA Microfabrication Instructional Laboratory (Microlab), which is discussed in more detail below. A week-by-week breakdown of the material covered in the laboratory-course lectures and lab sessions is given below in Table 2.

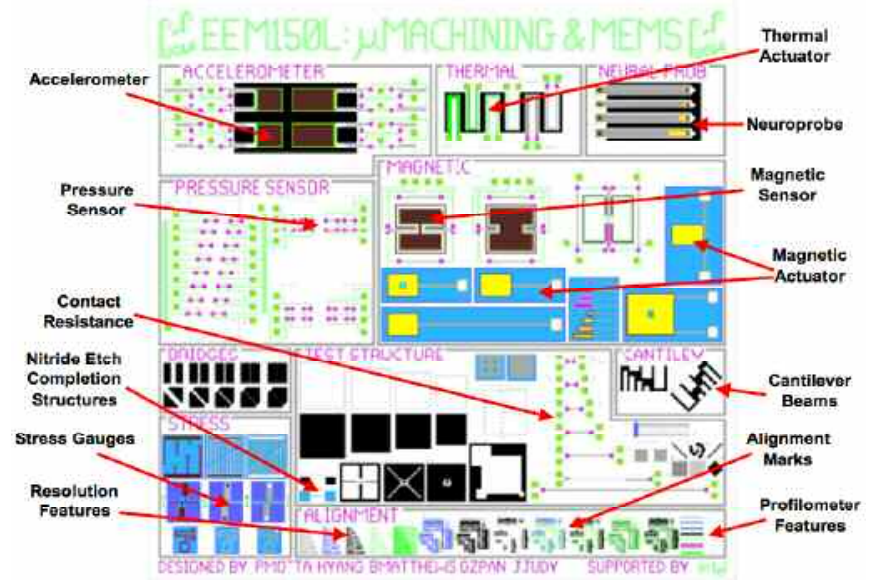

Fig. 1. Chip layout with devices and test structures indicated.

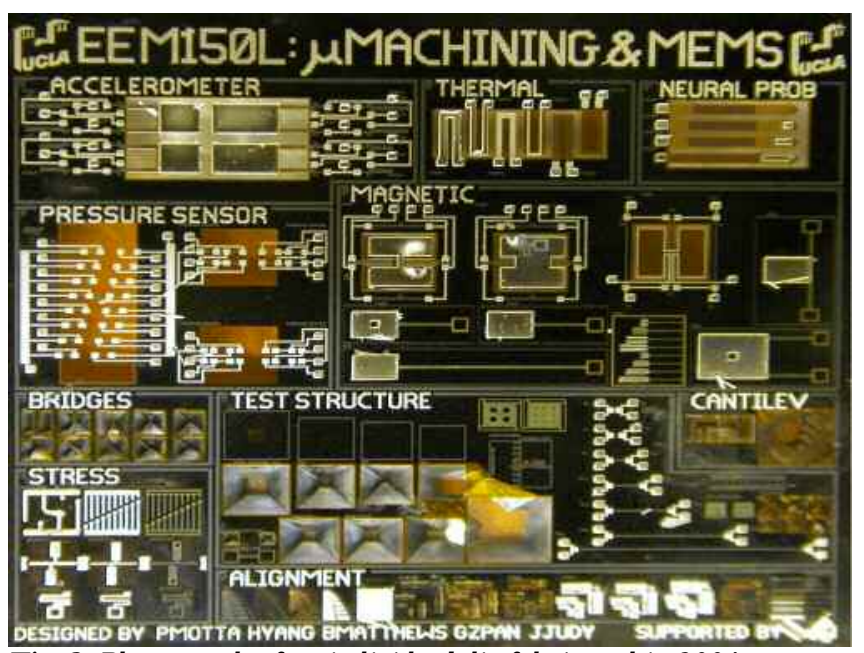

Fig. 2. Photograph of an individual die fabricated in 2004 ..

Table 2. Outline of the laboratory course.

\begin{tabular}{cl}
\hline Week & Laboratory Material (Lecture / Lab) \\
\hline Lec 0 & Admin, Overivew, Photolithography, Metrology \\
Lab 1 & $\begin{array}{l}\text { Safety Instruction, Walk Through, and } \\
\text { Introduction to Photolithography and Metrology }\end{array}$ \\
Lec 1 & Process Flow, LPCVD, Dry Etch, Profilometry \\
Lab 2 & Photolithography, Poly Dry Etch, Profilometer \\
Lec 2 & Alignment, LPCVD, Dry Etching, Spectrometry \\
Lab 3 & Mask Alignment, Nitride Dry Etch, Nanospec \\
Lec 3 & Front-to-Backside Alignment, Dry Etch, I-V Testing \\
Lab 4 & Dry Etch, Front-to-Backside Alignment, I-V Test \\
Lec 4 & Vacuum Systems, Evaporation, Lift-Off \\
Lab 5 & Dry Etch, Evaporation, Lift-Off \\
Lec 5 & Electroplating \\
Lab 6 & Electroplate Ni (Thin Through-Mask Plating)) \\
Lec 6 & Anisotropic Silicon Etch and Release \\
Lab 7 & Electroplate Ni (Over-Mask Plating), KOH Etch \\
Lec 7 & Device Physics, Testing, and Characterization \\
Lab 8 & Device Testing and Characterization \\
Lec 8 & Thanksgiving Holiday \\
Lab 9 & Device Testing and Characterization \\
Lec 9 & Device Physics, Testing, and Characterization \\
Lab 10 & Device Testing and Characterization \\
Lec 10 & Review Session and Class Photo \\
\hline
\end{tabular}




\section{E. Microfabrication Process Flow}

The objective of the design of the MEMS chip (Fig. 2) is to enable the demonstration of the fabrication of various MEMS microsensors and microactuators using a single fabrication process that takes 7 weeks to complete. The fabrication process (Fig. 3) consists of 7 photolithography steps, 6 etching steps, and 6 deposition steps. There are 16 chips per wafer, and in each chip contains over 100 devices: microsensors (accelerometers, pressure sensor, resonant magnetometers), microactuators (torsional magnetic microactuator [7], thermal microactuator), and microstructures (neural probe, stress test structures, bulk micromachining test structures). The diversity of devices fabricated was made very high to provide the students with an opportunity to test and become familiar with different types of microsensors and microactuators. However, since the process was obviously not optimized for any of the devices, the performance of each device is far less than that possible with an optimized process.

Starting with a single-side polished, $<100>$ oriented, $p$ type, $500 \mu \mathrm{m}$ thick silicon wafer, a $2-\mu \mathrm{m}$-thick low-stress silicon nitride is deposited using a low pressure chemical vapor chamber (LPCVD). Silicon nitride serves as both an insulator and as the mechanical layer. Next, a $1-\mu \mathrm{m}$-thick layer of LPCVD polycrystalline silicon (poly) is deposited and doped with boron. Polysilicon was chosen because it is piezoresistive (i.e., its resistance changes as a function of stress) and it is used as the sensing element in various devices on the chip. Polysilicon is also used as a sacrificial layer for other devices that are produced by surface micromachining. Once the polysilicon layer is patterned, the wafer is covered with another $1-\mu \mathrm{m}$-thick layer of low-stress silicon nitride. This layer also serves as a mechanical layer for surface micromachined structures and as the encapsulation to protect the resistors from the release etch. To enable the creation of bulk micromachined devices, openings are created on the backside of the wafer that are aligned to features on the front side. This is accomplished by using a front-to-backside alignment tool (Karl Suss MA-6). Once an opening is created in the upper nitride layer to reveal the polysilicon layer, electrical connection is made to the piezoresistors. A $0.1-\mu \mathrm{m}$-thick $\mathrm{Cr} / \mathrm{Ni}$ layer is then deposited by evaporation to form the seed layer for electrodeposition. Next a 5$\mu \mathrm{m}$-thick layer of low-stress nickel is electroplated to form the electrical interconnects. Since some devices require a large volume of metal to serve as the proof mass and as the magnetic torque element, another $25 \mu \mathrm{m}$ of electroplated nickel are deposited in specific regions. The release of the device is accomplished by etching exposed single-crystal silicon and polycrystalline silicon with potassium hydroxide $(\mathrm{KOH})$

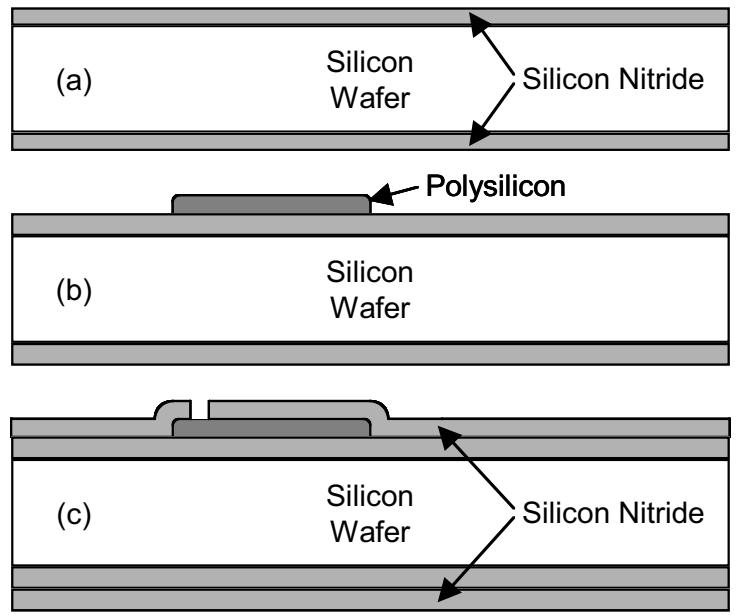

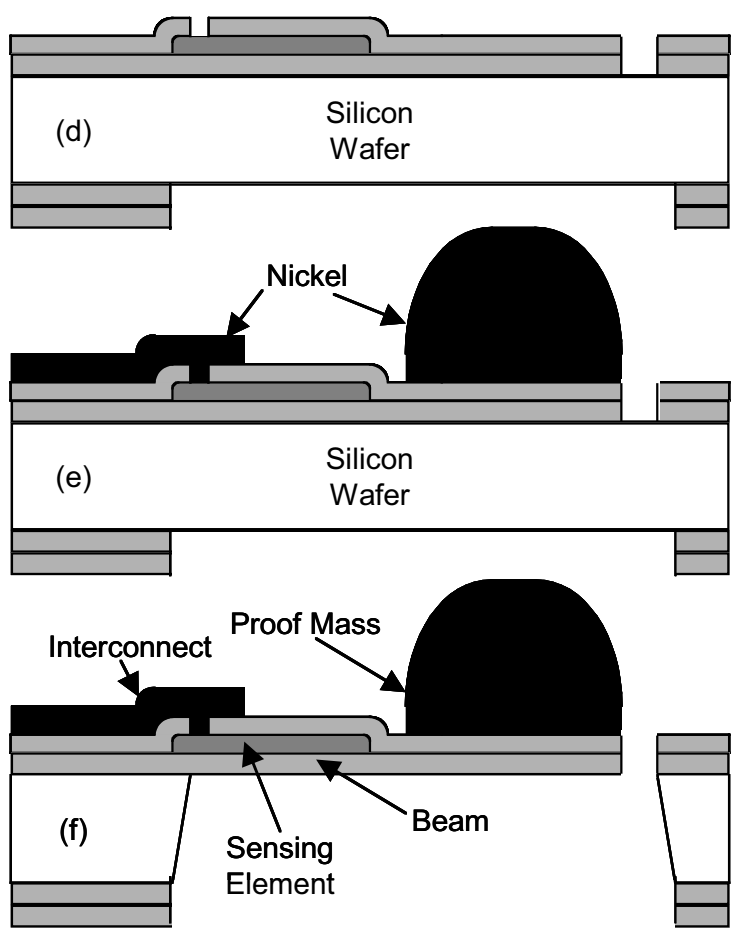

Fig. 3. Schematic diagram of the fabrication process flow followed in the hands-on laboratory course. With this process the student can produce a wide variety of surface-micromachined and bulkmicromachined microsensors and microactuators.

\section{F. UCLA Microfabrication Instructional Laboratory}

The Microlab instructional facility is a $306 \mathrm{~m} 2\left(3300 \mathrm{ft}^{2}\right)$ class 1000 cleanroom capable of processing $100 \mathrm{~mm}$ (4") wafers. It consists of a temperature-controlled and humidity controlled laboratory with integrated photolithography, wet etching, measurement, and lecture rooms. The lecture room is where students are instructed and quizzed about the microfabrication processes they perform. It also contains four probe stations with characterization equipment for device testing. The photolithography room (Fig. 4) contains two parallel processing lines for maximum throughput.

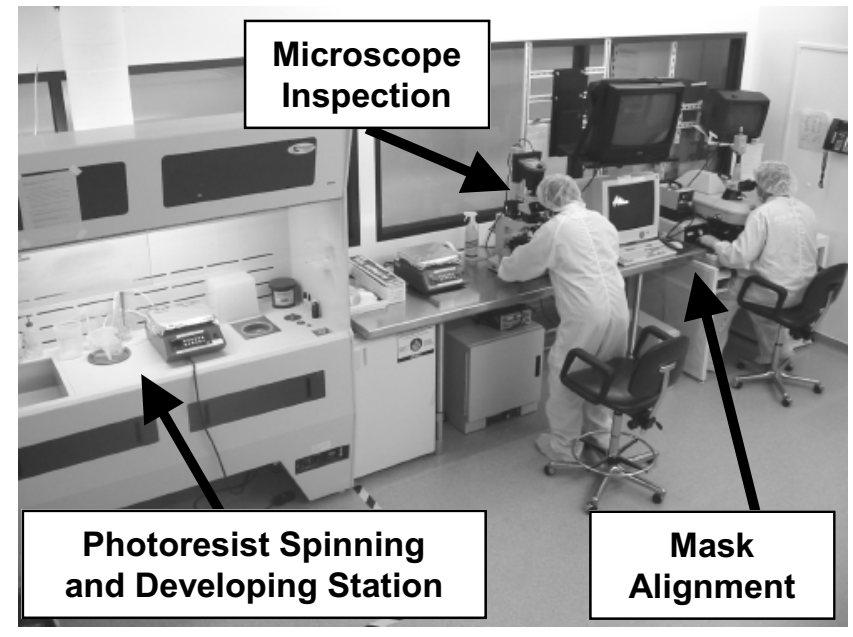

Fig. 4. Photograph of one of two parallel photolithography lines in the Microlab. Having two photolithography lines allows higher throughut and greater fault tolerance (i.e., if one line goes down the fabrication line may process wafers). 
Each processing line contains a wafer spinner, a Quintel mask aligner for UV exposure, a developing station and sink, and a microscope for inspection (Fig. 4). The etching room consists of two wet benches for wet etching processes. In addition, it contains a programmable spin dryer with 50 -wafer capacity, and a Tegal oxygen plasma-etching tool. The measurement room contains a Dektak profilometer and a Nanospec spectrometer. The microlab has a capability of teaching 180 students per year (i.e., 60 students/quarter).

\section{G. Testing}

Once released, the chips are separated from the wafer (i.e., V grooves fabricated into the wafer by design, allow it to be broken into chips without using a diamond saw) and then tested in one of four probe stations (Fig. 5). Students test all devices, but for brevity, only the accelerometer testing will be mentioned here.

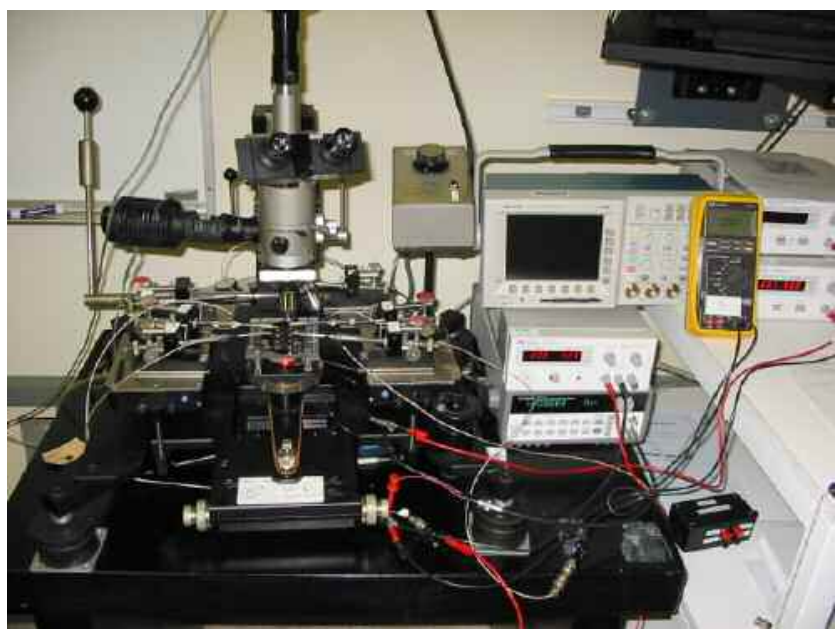

Fig. 5. One of four probe stations used for device testing.

The sensing in the accelerometer is done via doped polysilicon piezoresistors, which are laid out on the beams that support the proof mass and are integrated into a Wheatstone bridge circuit (Figs. 6 and 7). Piezoresistance was selected as the transduction mechanism due to its simplicity and robustness. To simulate acceleration, a magnetic field is applied to generate a magnetic torque on the ferromagnetic proof mass. The torque due to the magnetic field can then be related to the measured voltage across the Wheatstone bridge. By knowing the beam deflection, geometry, and material properties, the effective acceleration can be computed.

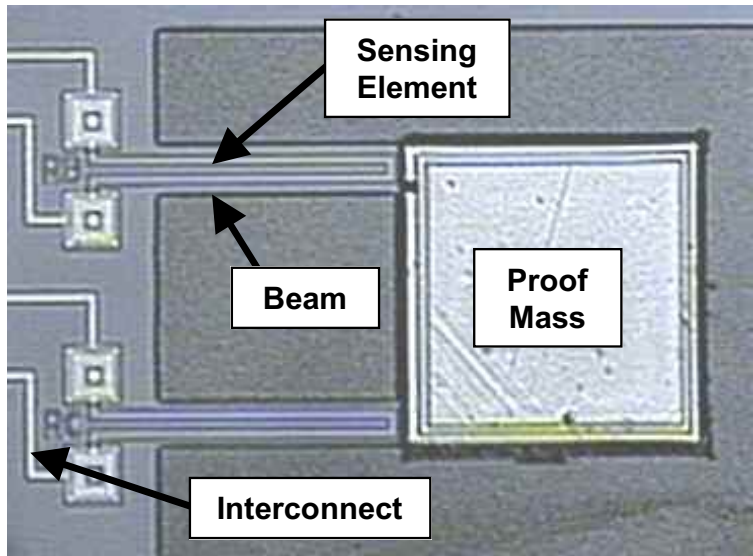

Fig. 6. Optical image of an accelerometer before being released by a bulk silicon etch. The sensing elements in the accelerometers and pressure sensors are piezoresistors due to their robustness.

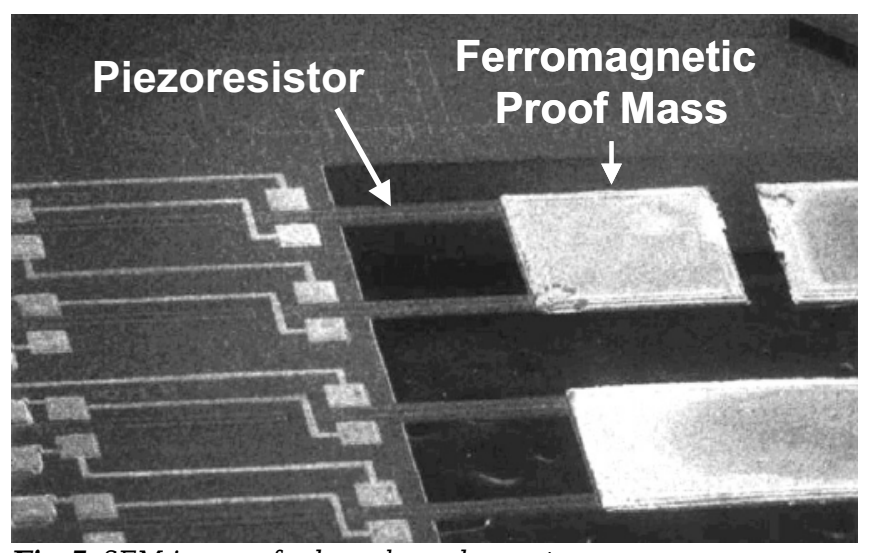

Fig. 7. SEM image of released accelerometers.

A plot of the deflection of the tip of the beam versus applied magnetic field is shown in Fig. 8. The error bars are due to experimental error, non-uniform electroplating of the proof mass, non-uniform applied magnetic field, etc. Despite the modest processing variability inevitable in any undergraduate laboratory, good device yield is obtained. The students are amazed, thrilled, and relieved that their hard work all term long has yielded functional devices.

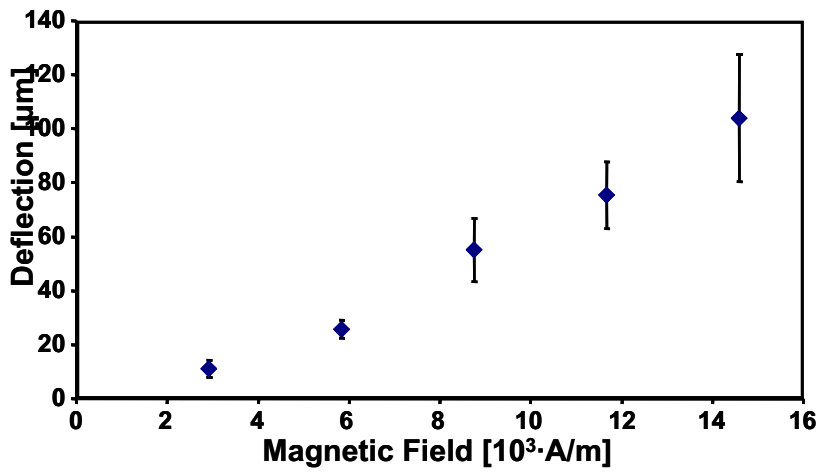

Fig. 8. Plot of deflection versus magnetic field applied show linear response.

\section{H. Trophies}

To provide the students with a symbol of their accomplishment, each year the students are presented with a trophy. This trophy consists of a acrylic block in which a single chip fabricated during their lab session is incased (Fig. 9). Students relate stories about bringing these trophies to job interviews to show what they have accomplished, explain what they did, and explain what MEMS are (if necessary).

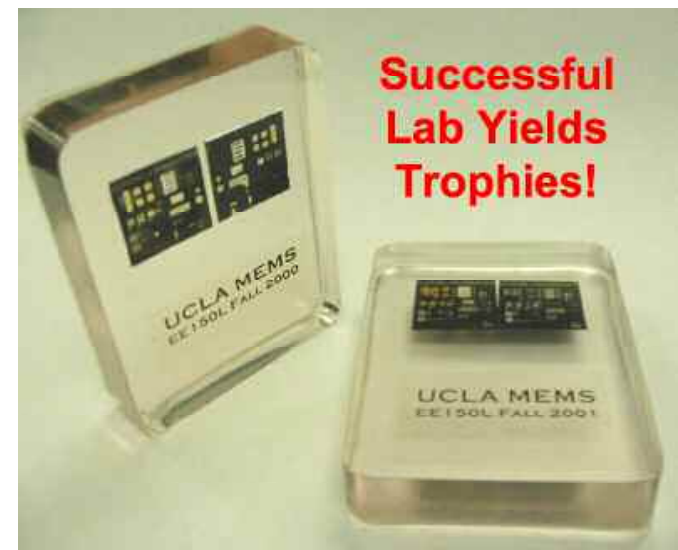

Fig. 9. Photography of the trophies presented to students who successfully complete the lab course. 


\section{Student Demographics}

The course was taught for the first time in the winter quarter of 1998 on an experimental basis, and a majority of the 12 enrolled students were not from electrical engineering (primarily mechanical engineering and chemical engineering). Figure 10 shows how the enrollment in the course has doubled each year it was taught until it was over subscribed in 2001 and the enrollment was capped at 64 students for laboratory safety reasons. In addition, Figure 10 illustrates how the diverse background of the students in the class has evolved from over time. Although students from chemical engineering (ChemE), civil engineering (CivE), computer science (CS), materials science (MatSci), and even neuroscience and medicine (MD) have taken the course, the majority of the students are in mechanical and aerospace engineering (MAE), electrical engineering (EE), and biomedical engineering (BME).

Figure 11 shows how the level of student in the class has changed over the years (1998-2002: combined course, 2003-2005: lecture-only course). Although these are undergraduate courses, they are clearly very popular with graduate students, as they have consistently made up the majority of the class.

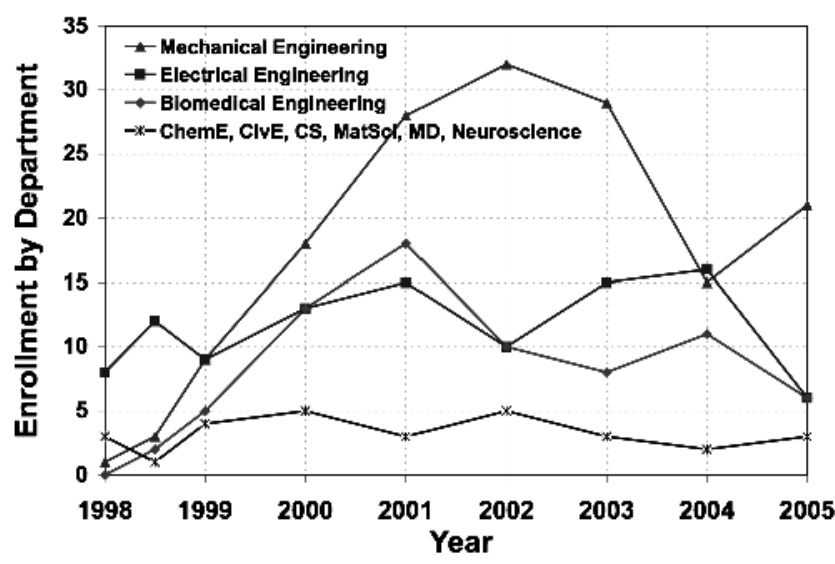

Fig. 10. Graph of enrolled student departmental affiliation during the years the course has been offered. Note: 1998-2002 correspond to the combined course and 2003-2005 to the lecture-only course.

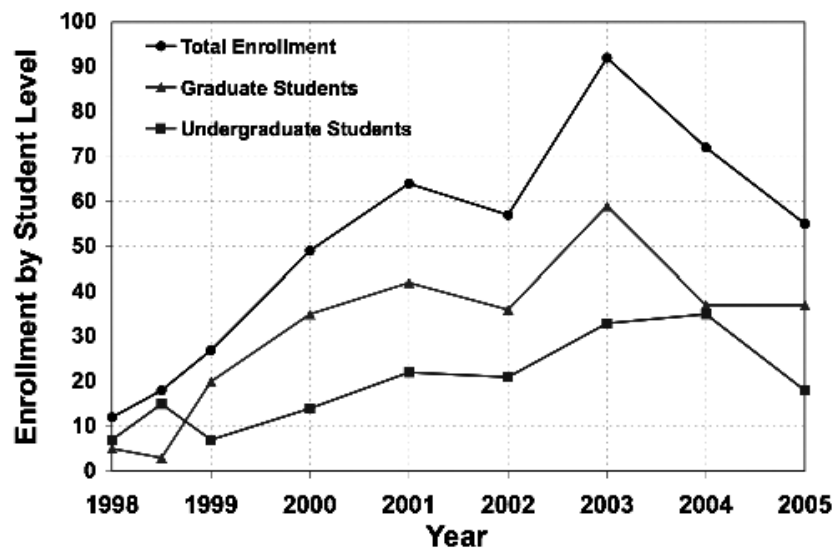

Fig. 11. Graph of enrolled student level (graduate and undergraduate) during the years the course has been offered. Note: 1998-2002: combined course and 2003-2005: lecture-only course.

\section{LESSONS LEARNED}

A 10-week course on micromachining and MEMS technologies has been designed that originally incorporated both a lecture component and a hands-on laboratory component. After three years, we learned that is would be far better to split these components into separate classes. That way many students, who only want to become familiar with MEMS and have no need to gain hands-on experience, would not take the scarce and valuable lab-session slots from a student that vitally needs the hands-on MEMS-fabrication experience provided. Although we have developed a microfabrication process that allows many different surface-micromachined and bulk-micromachined structures to be produced in parallel, an alternative approach with separate, shorter, and less complicated microfabrication processes should be considered.

\section{CONCLUSIONS}

Our undergraduate MEMS courses have been taught once a year since 1998. The enrollment has grown from 12, peaking at nearly 100 (lecture-only course), and settling between 60 and 70 students (lecture-only course). The laboratory course is capped at 60 students, and the enrollment is typically between 45 and 60 . Although most of the students are from electrical, mechanical, and biomedical engineering, students from many other disciplines, some even outside of engineering (e.g., neuroscience and medicine) have completed and benefited from the course. Despite being undergraduate-level courses, they is very popular with graduate students (66\% grads, 34\% undergrads). By completing the lecture-only course, the students gain an appreciation for MEMS and an understanding for their fabrication. By completing the laboratory course, the students gain hands-on experience with photolithography, isotropic and anisotropic wet etching, dry etching, physical and chemical vapor deposition, electroplating, MEMS release etching, stiction, and MEMS device testing. This set of undergraduate courses prepares students well to enter the graduate MEMS major field at UCLA offered in the MAE and EE departments [8], or to become active participants in the MEMS and microsystems industry.

\section{ACKNOWLEDGEMENTS}

This work was supported by an NSF Career Award (ECS 9876285). The authors would like to thank Grant Z. Pan and Eric Chang of the UCLA Microfabrication Instructional Facility for their technical support, and the teaching assistants Brian Matthews, Henry Yang, Chris Folk, and WanYu Wang, for their help developing the fabrication process.

\section{REFERENCES}

[1] R. C. Jaeger, Introduction to Microelectronic Fabrication, Addison Wesley, New York, 2001, ISBN: 0-2014-4494-1.

[2] S. Wolf and R.N. Tauber, Silicon Processing for the VLSI Era, Vol. 1: Process Technology, $2^{\text {nd }}$ Edition, Lattice Press, Santa Barbara, CA, 1999, ISBN: 0-9616-7216-1.

[3] M. Madou, Fundamentals of Microfabrication, CRC Press, Inc., Boca Raton, FL, 1997, ISBN 0-8493-9451-1.

[4] Richard S. Muller, Microsensors, New York: IEEE Press, 1991.

[5] Micromechanics and MEMS: Classic and Seminal Papers to 1990, W. Trimmer (Ed.), IEEE Press, New York, NY, 1996, ISBN 0-7803-1085-3.

[6] Gregory T. A. Kovacs, Micromachined Transducers Sourcebook, WCB/McGraw-Hill, 1998, ISBN 0-07-290722-3.

[7] Jack W. Judy, Richard. S. Muller, and Hans. H. Zappe, "Magnetic microactuation of polysilicon flexure structures," IEEE Journal of Microelectromechanical Systems, vol. 4, no. 4, pp. 162-169, 1995.

[8] Jack W. Judy et al., "Comprehensive MEMS Curriculum at UCLA," Tech. Dig. Solid-State Sensor and Actuator Workshop (Hilton Head '06), Hilton Head Island, SC (June 4$8,2006)$. 